\title{
Undiagnosed Borrmann type IV gastric cancer despite repeated endoscopic biopsies and PET-CT examination: A case report
}

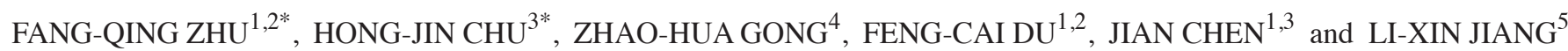 \\ ${ }^{1}$ First Department of Medical Oncology, Yantai Yuhuangding Hospital, Yantai, Shandong 264000; ${ }^{2}$ First Clinical College, \\ Dalian Medical University, Dalian, Liaoning 116000; ${ }^{3}$ Central Laboratory; ${ }^{4}$ Second Department of Medical Oncology; \\ ${ }^{5}$ Department of Gastrointestinal Surgery, Yantai Yuhuangding Hospital, Yantai, Shandong 264000, P.R. China
}

Received April 1, 2015; Accepted April 12, 2016

DOI: $10.3892 / \mathrm{ol} .2016 .4763$

\begin{abstract}
Borrmann type IV gastric cancer is a particular histological type of carcinoma, which has the characteristic of diffused infiltration that invades the entire stomach, resulting in the thickening and stiffness of the stomach wall. Borrmann type IV gastric cancer is known for the difficulty of detecting tumor cells in endoscopic biopsy specimens. This is crucial in obtaining the pathological results to make a therapeutic decision. The case reported in the present study was highly suspected to be Borrmann type IV gastric cancer according to the clinical manifestations and gastrointestinal barium meal examinations, but demonstrated negative results in multiple endoscopic biopsies and positron emission tomography-computed tomography (PET-CT) examination. The patient was discharged as no affirmative diagnosis was specified. Two weeks after discharge, the patient was administered to another hospital under emergency treatment due to frequent urination. Cystoscopy examination revealed marked thickening of the right bladder wall over a large area. Biopsy specimens were sampled. Pathological consultation suggested a gastrointestinal original of the lesion, which was most likely poorly differentiated gastric adenocarcinoma with neuroendocrine metastasis to the bladder.
\end{abstract}

Correspondence to: Dr Jian Chen, First Department of Medical Oncology, Yantai Yuhuangding Hospital, 20 Yudong Road, Yantai, Shandong 264000, P.R. China

E-mail: chenjianyt@163.com

Dr Li-Xin Jiang, Department of Gastrointestinal Surgery, Yantai Yuhuangding Hospital, 20 Yudong Road, Yantai, Shandong 264000, P.R. China

E-mail: jianglixin1969@hotmail.com

*Contributed equally

Key words: Borrmann type IV gastric cancer, endoscopic biopsy, positron emission tomography-computed tomography, diagnosis

\section{Introduction}

Borrmann type IV gastric carcinoma is a diffused type of gastric cancer (1) that presents with thickening and stiffening of the gastric wall as a result of invasive infiltration of at least a third of the circumference of the stomach (2). In 1858, Brinton first coined the term linitis plastica, which is also referred to as cirrous carcinoma in English literature, to describe infiltrative gastric carcinoma invading throughout the stomach (3). Approximately 10 to $15 \%$ of all gastric adenocarcinomas are considered to be Borrmann's type IV (4). This type is associated with poor prognosis as the disease is often diagnosed at an advanced stage. This may be due to difficulties in detecting the presence of gastric carcinoma under endoscopic inspection, as there is usually no ulceration or elevation appearing on the mucosal surface at the early stage of this malignancy (5). In addition, F-18 fluorodeoxyglucose positron emission tomography (FDG-PET) is of limited use in this lesion as primary gastric tumors are generally not susceptible to this radiopharmaceutical (6). This study reports the case of a patient suspected of having Borrmann type IV gastric carcinoma but for whom repeated endoscopic biopsies and positron emission tomography-computed tomography (PET-CT) examinations failed to confirm the diagnosis. Written informed consent was obtained from the patient.

\section{Case report}

Patient presentation and history. A 54-year-old male patient presented to Yantai Yuhuangding Hospital affiliated to Qingdao University (Yantai, China) on October 31, 2014, following 3 months of recurrent abdominal distention after eating. Gastric lesions were suspected. The patient had no notable medical or family history. He had been previously admitted to a local hospital due to the complaint of stomach discomfort, and had received two gastroscopy examinations. Gastroscopy had revealed erosive gastritis, but there was no apparent ulceration or other lesions indicating tumors. The biopsy results had revealed chronic gastritis with erosion of the corpora ventriculi. An upper gastrointestinal barium 
meal examination was then performed, and the examination results suggested the possibility of infiltrating gastric cancer. An abdominal contrast-enhanced CT scan was therefore performed, which revealed a thickened stomach wall of the gastric body. Gastric cancer was considered and the patient was referred to our hospital for further diagnosis and treatment.

Examinations and diagnosis. Physical examination upon admission revealed no anemia (via conjunctival pallor examination), jaundice or pulmonary abnormalities. The abdomen of the patient was bulging on palpation, with tenderness of the subxiphoid area. There was no pretibial edema, and no palpable abdominal mass or superficial lymph nodes. Blood tests revealed no abnormality of biomarkers including $\alpha$-fetoprotein, carcinoembryonic antigen, or tumor markers CA72-4 and CA19-9. A third gastroscope biopsy was later conducted, which only revealed chronic inflammation of gastric body mucosa (Figs. 1 and 2). The patient was suspected by the multidisciplinary discussion team of having Borrmann type IV gastric cancer. Another deep biopsy was performed with endoscopic ultrasound guidance, which revealed mucosal hyperemia of the fundus of the stomach and roughened mucosa in the corpus (Fig. 3). There was also marked thickening of the gastric wall ( $\sim 8 \mathrm{~mm}$; Fig. 4), low echo contrast, and loss of peristaltic movement. Endoscopic ultrasonography of the thickened stomach wall indicated features of typical changes of malignant lesions. Ten tissue cores were sampled by biopsy forceps in the thickened stomach wall. However, no tumor cells were identified by pathological analysis, so the diagnosis of chronic inflammation of the gastric mucosa was retained (Fig. 5). In order to exclude the possibility of gastric carcinoma, a PET-CT scan was requested. Examination revealed slight thickening of the lesser curvature wall in the proximal gastric angle region, and splenomegaly with normal $18 \mathrm{~F}$-fluorodeoxyglucose (18F-FDG) uptake (Fig. 6). PET-CT failed to identify malignant transformations. On November 12, the patient underwent a second upper alimentary tract barium meal examination, with the result suggesting possible infiltrative gastric carcinoma. Still, no definitive diagnosis was given. The patient was discharged at this point.

Two weeks later, he was admitted to another hospital due to frequent urination. Cytoscopy examination confirmed apparent thickening of a large area of the right bladder wall. The biopsy was sampled and immunohistochemical examination was performed. Consultation with the pathology department of Yuhuangding Hospital concluded that malignant cells were present in the interstitial tissue of the bladder (Fig. 7). Immunohistochemical examination revealed positive staining for $\operatorname{Syn}(+), \mathrm{CK}(+)$ and negative staining for $\mathrm{LCA}(-)$, SPA(-), CDX2(-) and CK20(-), which indicated a gastrointestinal origin of the pathology; specifically poorly differentiated gastric adenocarcinoma with neuroendocrine metastasis to the bladder. Therefore, in combination with the medical history, physical examination and auxiliary examination during hospitalization, the patient was diagnosed as having Borrmann type IV gastric cancer, and the histological type was confirmed to be gastric poorly differentiated adenocarcinoma with neuroendocrine metastasis.

\section{Discussion}

The classification of advanced gastric cancer (ADC) into four distinct types was introduced by Borrmann in 1926; these remain the standard criteria in cancer diagnosis $(7,8)$. Borrmann's type IV gastric cancer is characterized by a unique morphology both macroscopically and microscopically (9). Gastric linitis plastica is a diffuse infiltrative type of cancer in which the malignant cells invade a large area of the stomach, causing thickening and stiffening of the stomach wall (10).

The majority of patients presenting with symptoms are diagnosed at an advanced stage due to the limited sensory system of the stomach. Histopathological findings are essential to the diagnosis and therapeutic decision-making in Borrmann type IV gastric cancer. Histopathological diagnosis is the diagnostic gold standard of Borrmann type IV gastric cancer required by medical ethics and practice guidelines. However, tumor cells individually invade the submucosa without prominent ulcers or masses on the mucosal surface in the early stage. Early diagnosis of Borrmann's type IV cancer is difficult (9). Endoscopic biopsy demonstrated 90-98\% sensitivity in the detection of gastric cancer; however, in the case of Borrmann type IV AGC, not only detection of the tumor but also diagnosis of the specific type of tumor is documented as being difficult with gastroscopy. In addition, the success rate in correctly diagnosing the malignancy via endoscopic biopsy is also significantly lower in Borrmann type IV AGC compared with other Borrmann types. The unsatisfactory performances of gastroscopy and combined biopsy reflect the complex submucosa origination and unique morphological features of Borrmann type IV cancer. Infiltration predominantly occurs in the submicosal or muscular area, and is often associated with no apparent ulceration or elevation on the mucosal surface, which creates difficulty in endoscopic observation. Moreover, tumor cells are often widely dispersed within a dense fibrous stroma as a result of desmoplastic reaction that spares the mucosal layer, which may also influence pathological analysis of a small biopsy that may only contain the normal mucosal layer (11).

PET has been used to diagnose and monitor cancer lesions for many years. FDG is the most commonly used radiopharmaceutical, which uses the glucose metabolic path for diagnosing cancer. The increased metabolism of FDG is a characteristic of numerous cancer types. There are certain other radiotracers that have been proven useful in addition to 18F-FDG (12). FDG uptake is closely related to tissue property, rather than being specific to one type of malignant neoplasm. Therefore, attempts to use PET-CT with FDG in gastric cancer diagnosis may be unsatisfactory (12). Kawamura et al (13) reported that the expression level of GLUT1 protein in stomach carcinomas was $30 \%$ (128/617), and no expression was observed in 50 samples of tubular adenomas of the stomach; its expression in signet ring cell carcinoma and mucinous adenocarcinoma was relatively low, at $2 \%$ and $6 \%$, respectively. Among the other histological types, papillary adenocarcinoma (44\%) demonstrated slightly higher GLUT1 expression levels than the tubular $(32 \%)$ or poorly differentiated adenocarcinoma type (28\%) (14). In addition, tests for Borrmann type IV gastric cancer often present a false negative result due to the abounding mucin content. It should also be distinguished from 


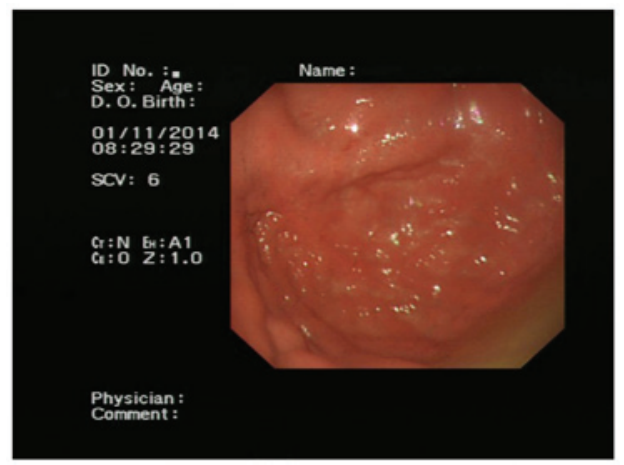

Figure 1. A third gastrofiberscopy revealed mucosal hyperemia in the gastric fundus and roughened mucosa in the gastric corpus.

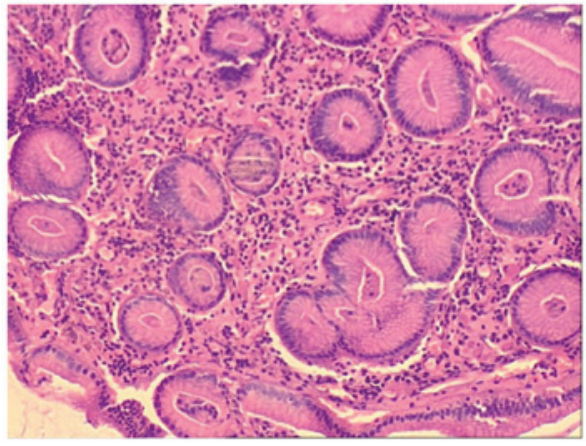

Figure 2. Superficial biopsy specimen revealed chronic inflammation of gastric body mucosa. Malignant cells were not observed. Hematoxylin and eosin staining; magnification, $\mathrm{x} 100$.

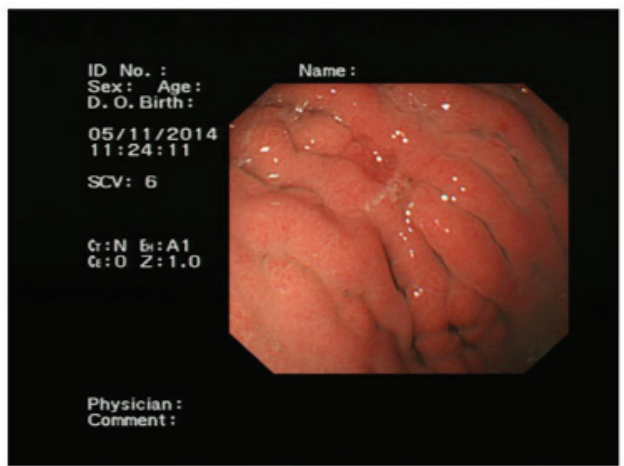

Figure 3. A fourth gastrofiberscopy revealed mucosal hyperemia in the fundus and roughened mucosa in the corpus.

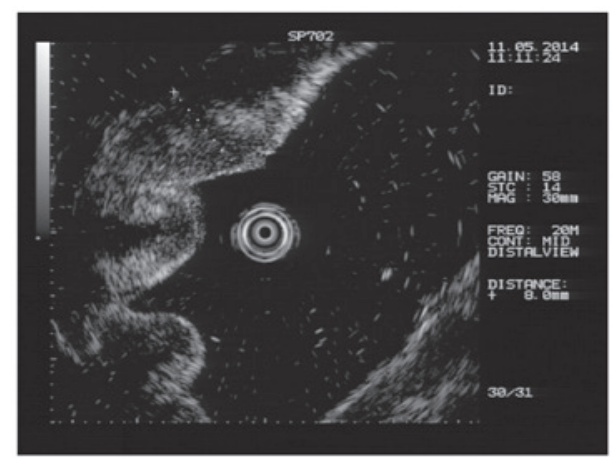

Figure 4. Endoscopic ultrasonography revealed that the gastric wall was significantly thickened by approximately $8 \mathrm{~mm}$.

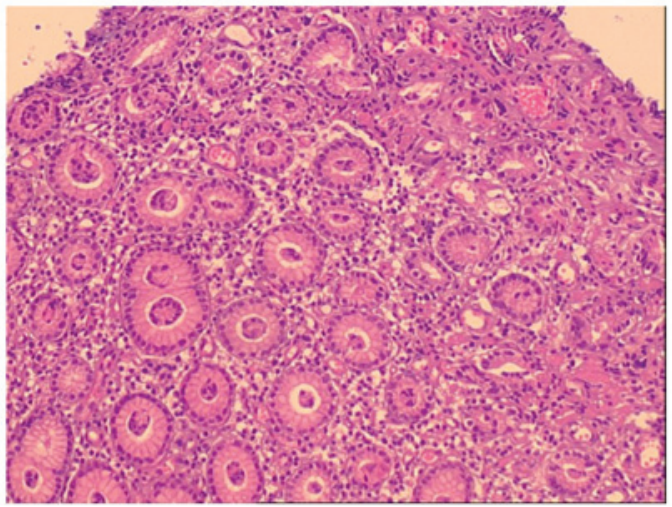

Figure 5. Deeper biopsy revealed chronic inflammation of the gastric mucosa. Cancer cells were not detected. Hematoxylin and eosin staining; magnification, $\mathrm{x} 100$.

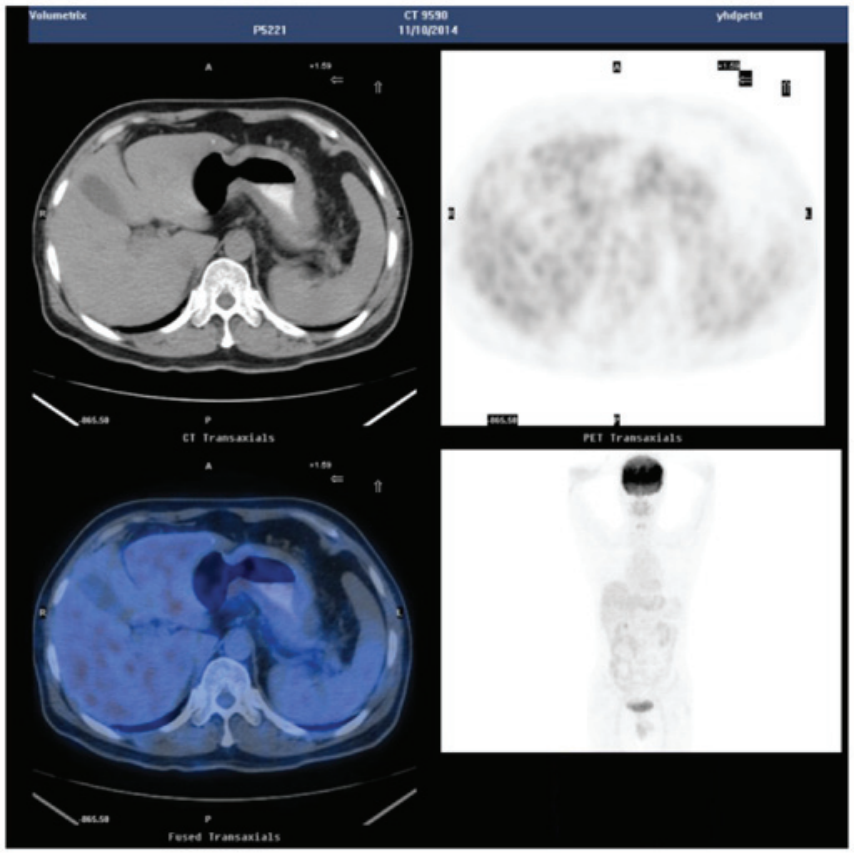

Figure 6. 18F-fluorodeoxyglucose (18F-FDG) positron emission tomography-computed tomography revealed slight thickening of the lesser curvature stomach wall in the proximal gastric angle region with normal 18F-FDG uptake and splenomegaly.

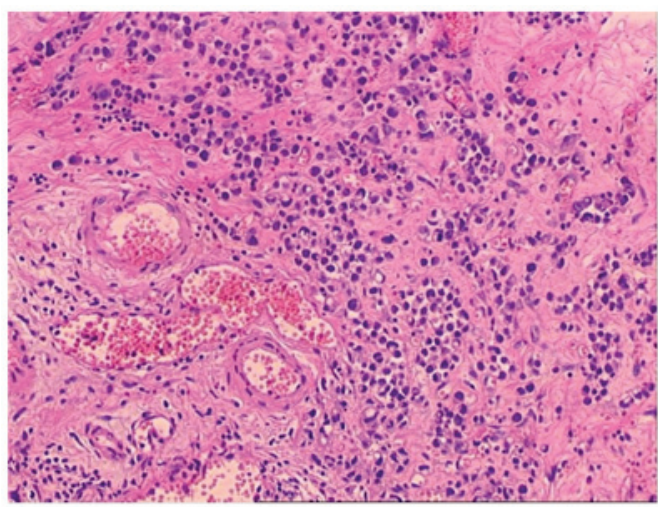

Figure 7. Biopsy specimen from the bladder revealed the presence of tumor cells in the interstitial tissue indicating the pathological diagnosis of poorly differentiated gastric adenocarcinoma with neuroendocrine metastasis to the bladder. Hematoxylin and eosin staining; magnification, x100. 
mucinous adenocarcinoma, signet ring cell carcinoma and poorly differentiated adenocarcinoma, which are also low in 18F-FDG uptake (15). Therefore, it would be inappropriate to confirm a diagnosis simply based on high FDG uptake, as this could also occur in gastritis or stomach ulcers (15).

In summary, accurate preoperative diagnosis of Borrmann type IV gastric cancer is extremely difficult, usually due to its distinctive infiltration pattern along the submucosal layer. Multiple gastroscopic biopsy and deep biopsy under radiological guidance that reaches the proper area of the lesion, are essential for the diagnosis of Borrmann type IV gastric cancer. The utility of 18F-FDG in PET-CT is limited in the diagnosis of gastric cancer; further evaluation of 18F-FDG uptake in gastric cancer is required.

\section{Acknowledgements}

This study was supported by the National Natural Science Foundation of China (grant no., 81071758), Shangdong Science and Technology Development Project (grant no., 2015GSF118142), the Natural Science Foundation of Shandong Province Joint Programme (grant no., ZR2015HL069) and Yantai Yuhuangding Hospital Initiative Foundation for Young Scientist (grant no., 201402).

\section{References}

1. Lauren P: The two histological main types of gastric carcinoma: diffuse and so-called intestinal-type carcinoma. An attempt at a histo-clinical classification. Acta Pathol Microbiol Scand 64: 31-49, 1965.

2. Pedrazzani C, Marrelli D, Pacelli F, Di Cosmo M, Mura G, Bettarini F, Rosa F, de Manzoni G and Roviello F: Gastric linitis plastica: Which role for surgical resection? Gastric Cancer 15 : 56-60, 2012

3. Moreaux J, Barrat F and Msika S: Linitis plastica of stomach. Study of 102 cases surgically treated. Results of the surgical treatment. Chirurgie 112: 485-492, 1986 (In French).
4. Accetta AC, Manso JE, Mello EL, Paiva RK, Castro Ldos S and Accetta P: Type IV Borrmann gastric adenocarcinoma: analysis of curative resection results. Rev Col Bras Cir 38: 237-244, 2011 (In English and Portuguese).

5. Yook JH, Oh ST and Kim BS: Clinicopathological analysis of Borrmann type IV gastric cancer. Cancer Res Treat 37: 87-91, 2005.

6. Sampath S, Harisankar CN, Bhattacharya A, Gupta R and Mittal BR: F-18 Fluorodeoxyglucose positron emission tomography/computed tomography in the staging of linitis plastica caused by primary gastric adenocarcinoma. World J Nucl Med 12: 67-69, 2013.

7. Borrmann R: Geschwulste des margens. In: Handbuch Spez Pathol Anat und Histo. Henke F and Lubarsch O (eds). 1st edition. Springer-Verlag, Berlin, Germany, pp864-871, 1926 (In German).

8. Borchard F: Classification of gastric carcinoma. Hepatogastroenterology 37: 223-232, 1990 .

9. Yokota T, Teshima S, Saito T, Kikuchi S, Kunii Y and Yamauchi H: Borrmann's type IV gastric cancer: clinicopathologic analysis. Can J Surg 42: 371-376, 1999.

10. Jafferbhoy S, Shiwani $\mathrm{H}$ and Rustum Q: Managing gastric linitis plastica: keep the scalpel sheathed. Sultan Qaboos Univ Med J 13: 451-453, 2013.

11. Kim JI, Kim YH, Lee KH, Kim SY, Lee YJ, Park YS, Kim N, Lee DH, Kim HH, Park do J and Lee HS: Type-specific diagnosis and evaluation of longitudinal tumor extent of borrmann type IV gastric cancer: CT versus gastroscopy. Korean J Radiol 14: 597-606, 2013.

12. Staniuk T, Zegarski W, Małkowski B, Jankowski M, Klag M and Pietrzak T: Evaluation of FLT-PET/CT usefulness in diagnosis and qualification for surgical treatment of gastric cancer. Contemp Oncol (Pozn) 17: 165-170, 2013.

13. Kawamura T, Kusakabe T, Sugino T, Watanabe K, Fukuda $\mathrm{T}$, Nashimoto A, Honma K and Suzuki T: Expression of glucose transporter-1 in human gastric carcinoma: Association with tumor aggressiveness, metastasis, and patient survival. Cancer 92: 634-641, 2001.

14. Małkowski B, Staniuk T, Srutek E, Gorycki T, Zegarski W and Studniarek M: (18F)-FLT PET/CT in patients with gastric carcinoma. Gastroenterol Res Pract 2013: 696423, 2013.

15. Song W, Chen CY, Xu JB, Ye JN, Wang L, Chen CQ, Zhang XH, Cai SR, Zhan WH and He YL: Pathological diagnosis is maybe non-essential for special gastric cancer: case reports and review. World J Gastroenterol 19: 3904-3910, 2013. 